\title{
Generation of thermal and pseudo-thermal plasmas
}

\author{
J.V.R. Heberlein \\ Department of Mechanical Engineering, University of Minnesota, Minneapolis, MN.
}

\begin{abstract}
From a review of the thermal plasma properties advantageous for materials processing, requirements are derived for thermal plasma generators. The generators are classified according to power conversion mechanism and the cathode electron emission, and designs for specific applications are discussed within each classification. Recent unconventional approaches to thermal plasma generation are also included.
\end{abstract}

\section{INTRODUCTION}

Thermal plasmas are characterized by high electron densities (typically above $10^{22} \mathrm{~m}^{-3}$ ) and temperatures between 5000 and $50000 \mathrm{~K}$. By definition, thermal plasmas are in Local Thermal Equilibrium (LTE), i.e. they can be characterized by a single temperature value except for the radiation field. The two principal advantages of thermal plasmas for materials processing are (1) the choice of medium - any material can become a plasma, and (2) the unique properties associated with thermal plasmas, namely high energy density (high specific heat), high thermal conductivity, a variable electrical conductivity, and high emission of radiation. Numerous devices make use of these characteristics, including circuit breakers, lamps and plasma reactors for materials processing, and the specific method of generating the plasma has to be adapted to the specific application. In this paper, the emphasis will be on thermal plasma generation for materials processing. In this application, the plasma generators have essentially two functions: (1) the transformation of electrical energy into thermal or plasma energy, and (2) the transfer of the plasma energy to the material to be treated. In order to utilize the advantages of thermal plasmas, the energy transfer should take place at temperature levels above those which can be achieved by other means, i.e. usually above $3000 \mathrm{~K}$. At these temperatures, the energy transfer from the plasma to a solid or a cold reactant is associated with strong gradients of temperature, density and composition, and consequently with deviations from equilibrium. The term "pseudo-thermal" is chosen to describe plasmas which have boundaries incompatible with LTE plasma conditions, which is the case for most processing plasmas. Therefore, the non-equilibrium boundary region surrounding the plasma is included in the consideration of the plasma generation.

In the next section, the characteristics of thermal plasmas will be discussed, leading to a classification of thermal plasma generators. A section on discharge stability is followed by a description of representative plasma generator designs for specific applications. Description of some unconventional approaches to thermal plasma generation conclude the paper.

\section{THERMAL PLASMA CHARACTERISTICS AND GENERATOR CLASSIFICATION}

Although there are several ways of generating a thermal plasma, I will concentrate in this paper on the generation by electric discharges. In a discharge, the plasma is generated by a current flowing in a partially or fully ionized gas, dissipating sufficient energy to keep the gas ionized and conducting. Although it is the electrons which pick up the energy from the applied electric field, the high number of collisions between electrons and other components of the plasma assure equlibrium distribution of the internal energy. The requirement for high collision frequencies sets a lower limit on the pressure of the thermal plasma, which is between 0.01 and $0.1 \mathrm{~atm}$.

Since in a thermal plasma the molecular components are mostly dissociated and atomic species are partially ionized, the energy transport occurs not only by transfer of kinetic energy as in ordinary gases, but includes transfer of the heat of dissociation and of ionization. This transport of reaction energy in addition to the fact that electrons transport heat more efficiently results in thermal conductivity values for thermal plasmas which are considerably higher than those of gases, and vary strongly with temperature. Therefore, even when the thermal plasma serves only as a medium to transfer heat, the plasma effects allow this transfer to occur more effectively. 
There are two types of discharges commonly used for the generation of thermal plasmas, the electric arc (dc or ac) and the high frequency (hf, or of for radio frequency) induction discharge. In the arc discharge, the plasma generating current flows from one electrode to the other through the plasma. The electrodes are in intimate contact with the plasma and experience the highest heat fluxes. The size of the plasma column is determined by the balance of electric power dissipation with heat loss by conduction, radiation and convection. For the same power input, a plasma gas with high thermal conductivity or with high radiative power loss values will have a smaller cross section, therefore higher energy densities. Energy densities increase for the following gases in the order in which they are listed: argon, nitrogen, helium, hydrogen.

Two principal uses of plasmas in materials processing are the bulk heating/ reacting of reactants and the energy transfer to surfaces. There are in principle three ways in which discharge plasmas can be provided for these tasks: (1) The material to be treated is passed directly through the discharge; in this case the material is exposed to the highest heating rates, and the power dissipation will adjust to the heating requirements and will only be limited by the plasma system design. (2) The plasma is extracted from the discharge region by a superimposed flow, and this plasma jet is used to treat the materials; mixing of the plasma jet with the material or with a cold environment will cool the plasma, thus limiting the amount of energy available for the treatment. Both, arc discharges and hf discharges can be used for these arrangements. (3) For the treatment of a metallic surface, an arc discharge can be transferred to the surface making it one of the electrodes; the highest surface heating rates and most efficient energy transfers are obtained in this way.

Plasma generators are classified according to the generation method as arc discharge (dc or ac plasma torch) or hf induction generators, and for the arc discharge generator according to the energy transfer mechanism as transferred arc if the material to be treated serves as one electrode, or as non-transferred arc plasma torch.

An additional classification method for the arc discharge generator is given by the cathode emission mechanism which characterizes the arc operation. The arc discharge requires emission of electrons from the cathode, and there are two basic mechanisms by which a surface can provide these electrons: (1) Thermionic emission from a surface heated to a temperature allowing the escape of sufficiently large numbers of electrons from the conduction band; typically temperatures above $3000 \mathrm{~K}$ are needed for this emission mechanism, and refactory metals like $\mathrm{W}$ are used to avoid bulk melting; addition of a low workfunction material such as $\mathrm{ThO}_{2}$ or $\mathrm{La}_{2} \mathrm{O}_{3}$ to the $\mathrm{W}$ will increase the number of electrons emitted at a given temperature. (2) Field emission of electrons due to high electric fields in front of the surface; this emission mechanism rarely provides a sufficient number of electrons for arc plasmas, but a combination of field with thermionic emission (TF emission), or with an emission mechanism where microscopic evaporation sites provide a partially ionized metal vapor, is the dominant provider of electrons with "cold cathodes" such as water-cooled copper. The principal characteristics of the emission mechanisms are listed in Table 1. The erosion rates are very low for cold cathodes at current levels below $2 \mathrm{kA}$, and increase strongly for higher currents. The erosion rate for $\mathrm{W}$ - cathodes are low but increasing with current up to approximately $10 \mathrm{kA}$, and increase strongly above this level. Graphite electrodes exhibit very high erosion rates, but usually the erosion product is used to provide a reducing atmosphere (consumable electrode compared to the non-consumable W - cathode). Cold cathodes can be used with oxidizing plasma gases, whereas the erosion rate of hot cathodes will increase drastically with the use of oxygen due to the formation of volatile oxides.

Table 1: Characteristics of Cathode Electron Emission Mechanisms

$\begin{array}{llll}\begin{array}{l}\text { Mechanism/ } \\ \text { Material }\end{array} & \begin{array}{l}\text { Surface } \\ \text { Temperature }\end{array} & \begin{array}{l}\text { Current } \\ \text { Density }\end{array} & \text { Current } \\ \begin{array}{l}\text { Thermionic (hot)/ } \\ \text { W + Th } \mathrm{O}_{2}\end{array} & >3000 \mathrm{~K} & 10^{7}-10^{8}{\mathrm{~A} / \mathrm{m}^{2}}^{2} & 1 \mathrm{~A}-10 \mathrm{kA} \\ \begin{array}{l}\text { Graphite } \\ \text { Cold cathode/ }\end{array} & >3000 \mathrm{~K} & 10^{7}-10^{8} \mathrm{~A} / \mathrm{m}^{2} & 5-100 \mathrm{kA} \\ \begin{array}{l}\text { Cu or Cu alloys, } \\ \text { steel }\end{array} & <2000 \mathrm{~K} & 10^{10}-10^{12} \mathrm{~A} / \mathrm{m}^{2} & 0.05-2 \mathrm{kA} \\ & & & \end{array}$




\section{PLASMA STABILITY}

Thermal plasmas are inherently unstable, and usually one has to take several steps to stabilize them.

Electrical instability of an arc derives from the fact that the arc discharge has a negative characteristic over a wide range of operating parameters, i.e. a decrease in voltage will lead to an increase in current which in turn will further decrease the voltage. For dc arcs, modern power supplies provide thyristor control of the current output obviating the use of series impedances. For ac arcs, series inductances can provide stabilization.

Thermal Instability of the plasma is caused by the fact that cooling of the plasma to the temperature of the surroundings will extinguish the plasma. Consequently, the electrical power supplied to the plasma has to exceed the energy requirement for heating the gases to temperatures which assure sufficient ionization for unimpeded current flow. For arc discharges, cooling of the plasma will lead to constriction of the discharge channel and to higher voltages, strongly cooled discharges need high voltage power supplies. High frequency discharges are usually more vulnerable to thermal instabilities.

Magneto-hydrodynamic (MHD) instabilities are encountered when the change in the self-magnetic field of an arc discharge due to a disturbance magnifies the effect of the disturbance. Most arc discharges have to be stabilized against MHD instabilities, and thermal plasma generator designs are using different stabilizing mechanisms. The most frequently used stabilizing effect is the cooling of the plasma when it deviates from the stable position, either by using a water-cooled solid wall (wall-stabilization) or by using a cold gas shield frequently in form of a swirl flow around the plasma (convection or vortex or swirl stabilization).

Arc restriking can occur when the arc is forced to increase its voltage by e.g. increasing its length. A cold flow parallel to the anode will move the arc-electrode attachment until the arc voltage has reached a value that upstream penetration of the cold gas boundary layer is energetically preferred (restriking of the arc). A constant arc motion with varying arc voltage and power dissipation may be the consequence.

Fluid-dynamic instabilities are observed when a plasma jet exits a nozzle into an cold gas environment with vastly different density and viscosity. The strong property gradients will amplify the turbulence in the boundary layer between the hot jet and the cold gas leading to large scale fluctuations.

HF induction plasmas have a stability criterion imposed by the skin depth determining the penetration of the hf electromagnetic field into the plasma. The consequence is a plasma frequency - size correlation which gives the range of stable operating conditions (ref. 1).

\section{PLASMA GENERATORS FOR SPECIFIC APPLICATIONS}

In this section, generator designs for specific applications are discussed for each of the plasma generator classes, together with recent developments in adapting the generators closer to the requirements. An overview of what generator types are used at what arc current, voltage and power level is given in Fig. 1 .

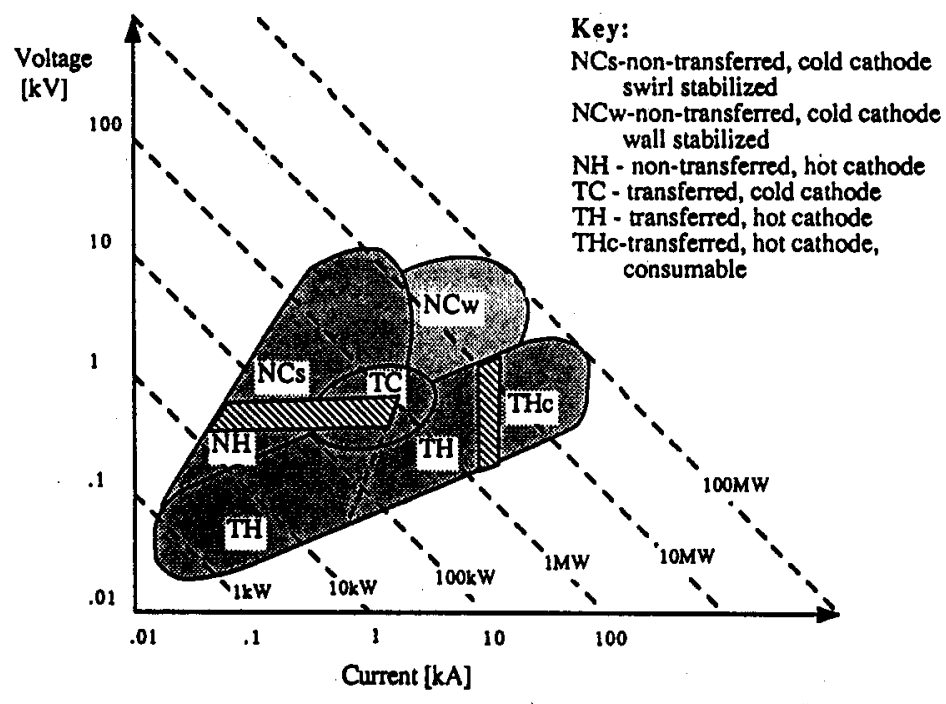

FIG. 1: Characteristics of Thermal Plasma Generator Types 
It is apparent that there is a strong overlap in operating conditions of transferred and non-transterred arc devices with hot cathodes at the lower power levels. However, cold cathode non-transferred torches extend the power range by going to higher operating voltages, whereas hot cathode transferred arc devices go to higher currents.

\section{Non-transferred hot cathode plasma torches}

This type of torch is one of the most widely used arc gas heaters for non-oxidizing gases. It is extensively used for plasma spraying, the high energy densities and velocities providing good heat and momentum transfer, as well as for plasma synthesis and for surface treatment. In a typical design, a thoriated tungsten rod cathode is positioned in the entrance of the cylindrical, water-cooled anode nozzle (see Fig. 2). The

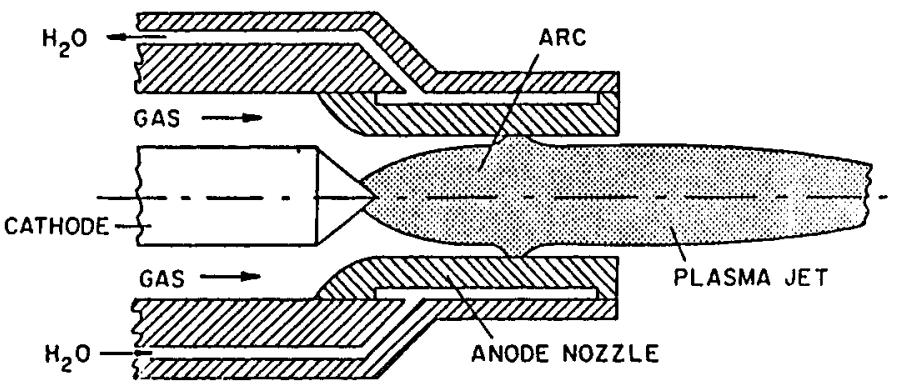

FIG. 2:

Schematic of Typical Non-Transferred Hot Cathode Type Plasma Generator for Spraying or Synthesis

plasma gas is introduced at the bottom of the cathode rod which is water-cooled. A swirl component of the plasma gas helps stabilize the cathode attachment and move the anode attachment to distribute the heat flux to the anode. For spray applications, the spray powder is injected into the plasma either through a hole in the anode nozzle wall or immediately outside the nozzle exit. The plasma has been well characterized in numerous studies,including measurements of temperature and velocity fields, arc and jet stability, and heat transfer to injected particulates. (references 2-6). The arc can display the restrike instability, and in most cases, the jet is strongly turbulent and frequently instable. In order to increase the jet velocity and obtain a more stable jet, expansion through a supersonic nozzle into a low pressure chamber is being used for advanced high quality coatings. Other developments include designs of hot anodes to reduce the restrike phenomenon and of converging nozzles to improve jet stability (see Fig. 3).

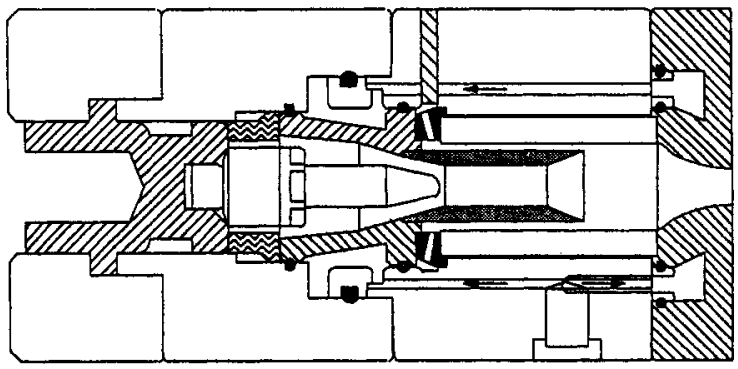

FIG. 3:

New Plasma Torch for Improved Jet Stability with Hot Anode Tube and Converging Nozzle

For plasma synthesis applications, the reactants are usually injected downstream of the nozzle exit, and mixing occurs in a confined channel flow. A more elegant and more energy efficient way of mixing the reactants with the plasma is accomplished by using multiple plasma jets converging at the spot where reactants are injected (references 7,8 ), or by injecting the reactants in a counterflow arrangement to the plasma jet (ref 9).

\section{Non-transferred, cold cathode plasma torches}

Several designs for such arc heaters exist and have been discussed in detail in ref.10. The arc is struck typically between two co-axial, cylindrical, water-cooled electrodes, the gas is introduced between the electrodes with a swirl component, and in most designs a magnetic field generated by a coil surrounding the electrodes provides additional motion of the electrode attachment spots thus reducing electrode wear (see Fig. 4). Major applications are in process gas heating for chemical or metallurgical processes, e.g. synthesis of $\mathrm{TiO}_{2}$, synthesis of $\mathrm{C}_{2} \mathrm{H}_{2}$, or firing of blast furnaces or cupolas to reduce coke and wind requirements (references 10-14).

The majority of these arc heaters are swirl stabilized, and high mass flow rates through the plasmas result in high gas heating efficiencies, higher arc voltages and lower enthalpy values than one obtains with hot cathode torches. However, the enthalpy values can be increased by going to longer arcs and even higher arc voltages (see Fig.5). 


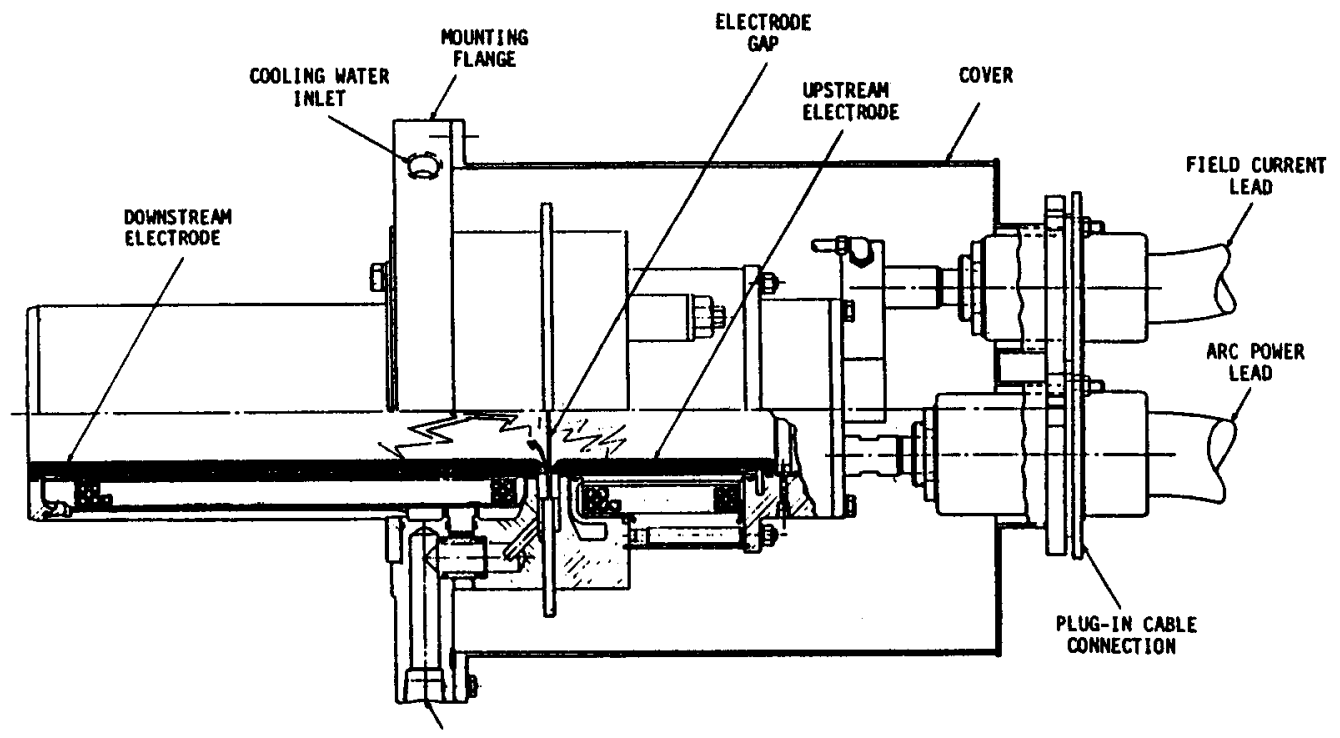

GAS INLET

FIG. 4: Cross section of swirl stabilized, non-transferred cold cathode plasma torch (with permission of Westinghouse Electric Corp.). The gas enters through the gap between the electrodes; magnetic field coils enhance arc rotation

The highest enthalpies can be achieved with low mass flow rates and wall stabilization. A development by NASA of an arc heater for re-entry simulation used $10 \mathrm{~m}$ long arcing channels, with a large number of water-cooled constrictor sections, insulated against each other to avoid series arcing (ref.15). An interesting cathode design using a series of parallel cathode rings allowed the total current to be pushed to $10 \mathrm{kA}$ without excessive erosion. Although this particular arc heater design is not suited for processing applications, the principle of including in a swirl stabilized arc heater insulated constrictor sections between the electrodes to increase the arc power and the plasma enthalpy has been used by several plasma torch manufacturers.

\section{Transferred arc, cold cathode torches}

A few manufacturers offer this type of plasma generator. Its major application is in the metal remelting and refining industry (see Fig. 6), although recent developments are proceding in adapting such generators to waste processing (ref. 16,17). Compared to hot cathode transferred arc devices, the cold cathode offers longer electrode life. Compared to non-transferred cold cathode torches, the transferred arc device allows considerably higher heat fluxes and energy transfer efficiencies.

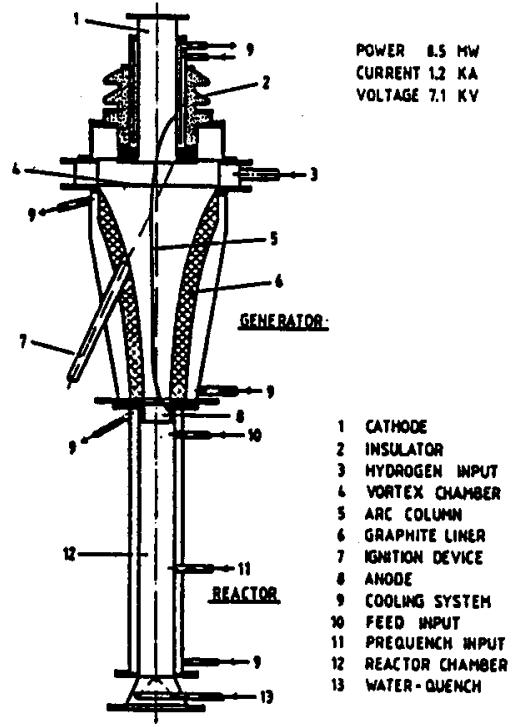

FIG. 5: Schematic of non-transferred cold cathode plasma torch with high are voltage (ref. 12 with permission of the author)

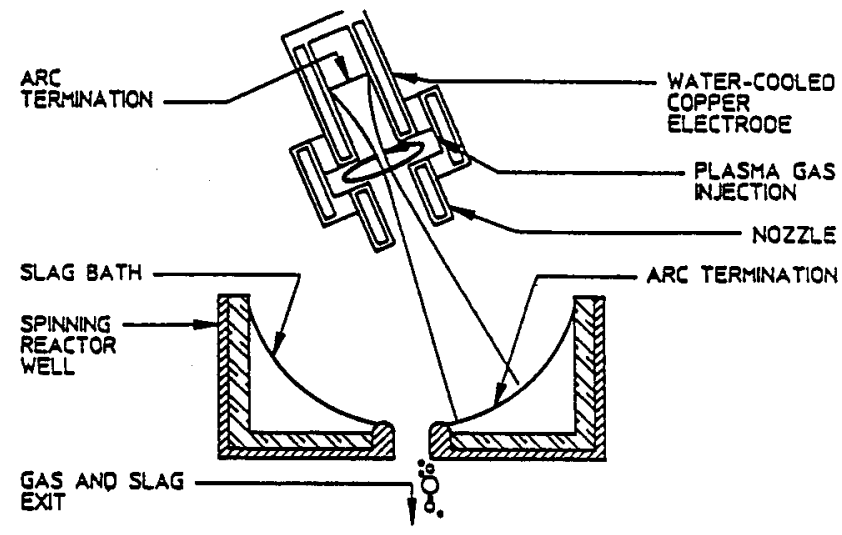

FIG. 6: Schematic of transferred arc cold cathode torch for remelting (ref. 16 with permission of the author) 


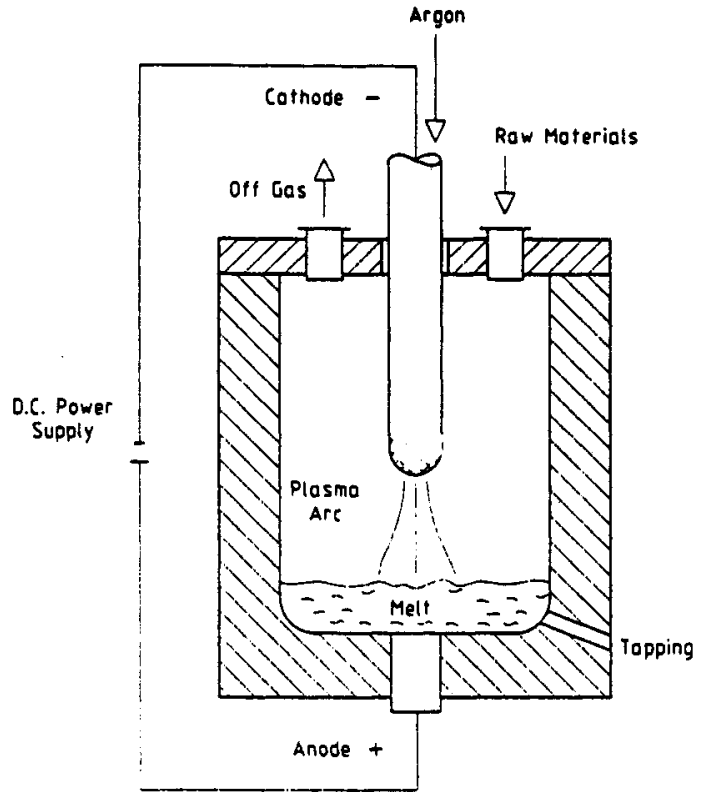

FIG. 7: Schematic of high power transferred arc reactor with consumable hot cathode

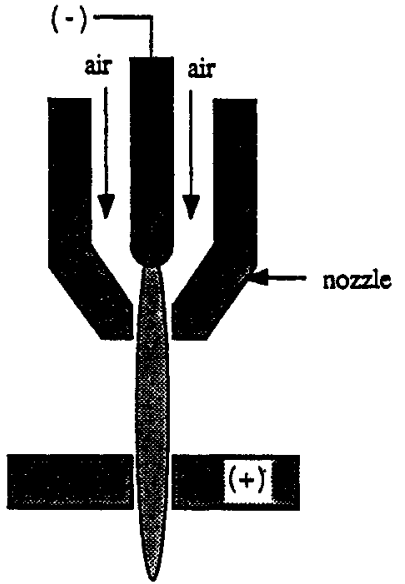

FIG. 8: Schematic of hot cathode transferred arc cutting torch

Transferred arc, hot cathode generators

These devices are being used over a wide range of powers, from $100 \mathrm{~W}$ to $100 \mathrm{MW}$. The main differences between different designs are the cathode material and the arrangement for the plasma gas introduction along the cathode (see Fig. 7). All of these type of generators are optimized with respect to the heat flux to the anode. The application in order of increasing power consumption are cutting (Fig 8), welding, metal remelting and refining, tundish and ladle heating, and metal melting and smelting (Fig. 7).

For applications requiring up to approximately $10 \mathrm{MW}$, non-consumable, i.e. mostly tungsten based cathodes are preferred. For higher current levels, the high erosion rates require use of consumable graphite electrodes. The arc is initiated by contact of the cathode with the metal and withdrawal to the desired position, or by using a pilot arc between the starter electrode and a ring shaped main electrode. The plasma gas is introduced through channels surrounding the rod cathode in low power applications, and additionally through a central bore in the cathode at high powers. (references 18, 19). In metal melting or waste processing applications using non-consumable electrodes, pre-heating of the charge can be accomplished by exposing the material to the arc heat before it falls into the anode bath. In high power applications, fines can be introduced through the central bore in the cathode.

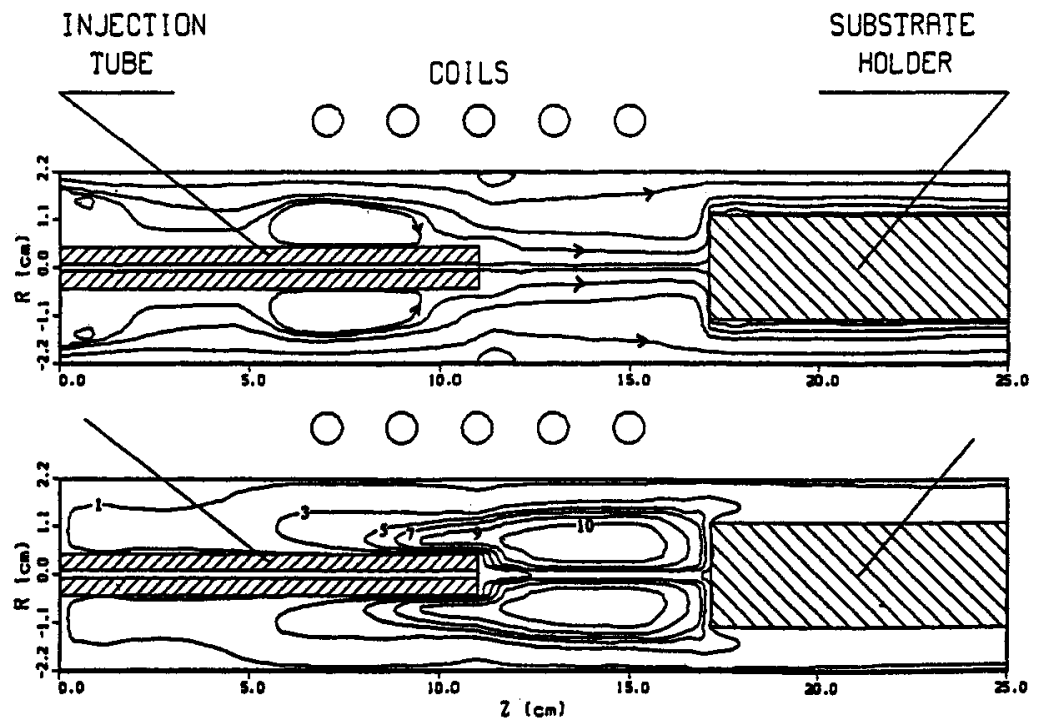

FIG. 9: High frequency plasma reactor for thermal plasma CVD with streamlines (top) and isotherms (bottom) indicated (ref. 21, with permission of the author) 
The recent developments have been concerned mostly with extension to higher powers in the metallurgical industry, using new electrode designs, and to adaptation of specific torch designs to new applications such as underwater cutting and destruction or consolidation of hazardous wastes.

\section{HF induction plasma torches}

These torches have been applied to plasma spraying and to plasma synthesis (ref.9, 20, 21). The main advantages are longer residence time of the material in a hot plasma zone and a more uniform temperature distribution. However, torches have not been scaled to beyond $1 \mathrm{MW}$ of power, and the energy conversion efficiency is relatively poor. Fig. 9 shows a schematic of a HF torch with calculated streamlines and isotherms.

\section{NEW APPROACHES}

Three unconventional ideas in plasma generation methods have been advanced in the past several years: a hybrid dc arc torch fired if plasma generator, and an atmospheric pressure microwave plasma torch developed at he University of Tokyo (ref. 22, 23), and extension of the surface wave mode microwave plasma generator to thermal plasmas (ref 24). The hybrid plasma torch extends the stable operating regime of an hf plasma reactor by preionizing the plasma gas using the jet of a dc non-transferred plasma torch directed towards the plasma generation region of the hf induction generator (see Fig.10). The consequence is that higher throughputs of reactants through the discharge can be achieved. The applications deriving potential benefits from this development are plasma synthesis and plasma waste destruction.

The atmospheric pressure microwave torch (Fig. 11) couples the microwave power from a waveguide to a pointed "electrode". A discharge between this electrode and a surrounding ring-shaped ground "electrode" has been used to generate a hydrogen plasma at one atmosphere without erosion of the "electrodes". The plasma generation is thought to occur through direct coupling of the microwave power to the plasma after the initial breakdown, and the "electrodes" provide only a field enhancement for breakdown and for anchoring the discharge.

The surface wave dicharge generates a plasma by a special mode of microwave propagation along an insulating surface. A "wave launcher" couples the microwave power to the plasma flow tube and initiates the surface wave plasma. Further extension of this concept to higher power levels may lead to a new generator for pseudo-thermal processing plasmas.
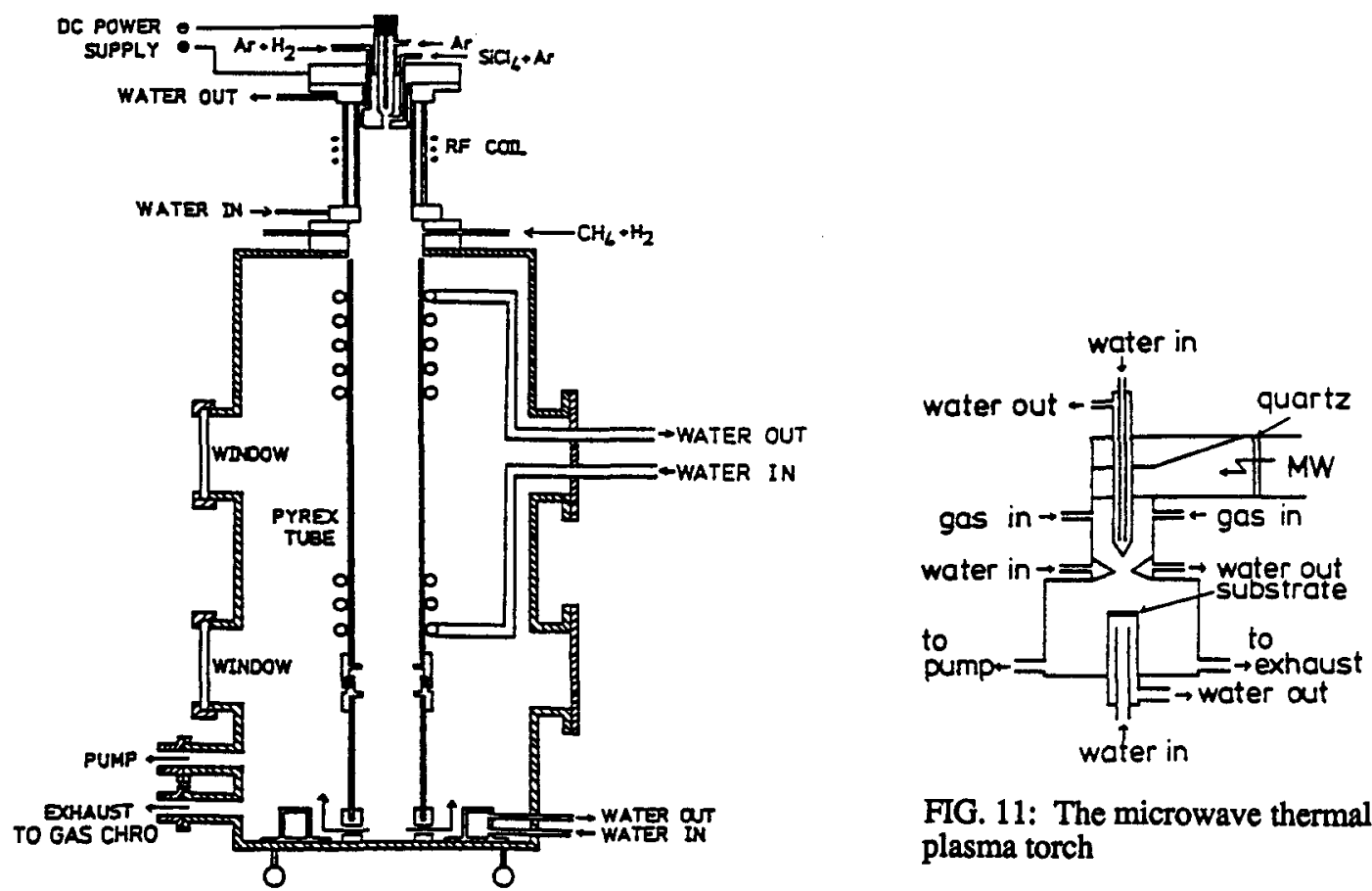

FIG. 11: The microwave thermal plasma torch

FIG. 10: The hybrid plasma reactor 


\section{CONCLUSIONS}

A review of the major applications of thermal plasma technology indicates that there are continued advances being made in the development of plasma generating devices. For the most part, these advances consist of extension of the generator designs to higher power levels by redesigning electrodes, or of adapting existing designs to new applications. However, some efforts point towards using unconventional designs offering specific advantages. With several new potential applications such as thermal plasma CVD of diamond or plasma destruction or consolidation of hazardous wastes being pursued, new developments of plasma generating devices offering increased energy utilization, improved stability and better reactant mixing and product uniformity would certainly be welcome.

\section{REFERENCES}

1. Plasma Technology in Materials Processing, J. Feinman ed., Iron and Steel Soc., Inc., Warrendale, PA., (1987).

2. M.I. Boulos, Pure and Applied Chemistry, 57, 1321-1352 (1985).

3. M. Vardelle, A. Vardelle, P. Fauchais, M.I. Boulos, A.I.Ch.E.J., 29, 236-243, (1983).

4. W.L.T. Chen, J. Heberlein, E. Pfender, Proc. 10th ISPC, Bochum, Germany (1991).

5. R. Spores and E. Pfender, Surface and Coatings Technology, 37,251 (1989).

6. E. Pfender, Plasma Chem. and Plasma Proc., 911 Suppl., 277 (1989).

7. M.G. Fey, T.N. Meyer, W.H. Reed, Proc. 4th ISPC, Zurich, Switzerland, 708 (1979).

8. R.M. Young and E. Pfender, Plasma Chem. and Plasma Proc., 2, 465 (1989).

9. P.C. Kong and Y,C. Lau, Pure and Applied Chemistry, 62, 1809 (1990).

10. S.L. Camacho, Pure and Applied Chemistry, 60 (5), 619-632, (1988).

11. A.P. George and A.L. Hare, Proc. 6th ISPC, M.I. Boulos and R.J. Munz, eds., Montreal, Canada, 144 (1983).

12. R. Muller, L. Kerker, G. Pross, C. Penckert, Proc. 8th. ISPC, K. Akashi and A. Kinbara, eds., Tokyo, Japan, 660 (1987).

13. J.V.R. Heberlein, W.J. Mellili, S.V. Dighe, W.H. Reed, Proc. Workshop on Industrial Plasma Applications, M.I. Boulos, ed., Pugnochiuso, Italy, 1 (1989).

14. Y. de Lassat de Pressigny, A. Butel, R. Bloise, R. Drelon, ibid., 103.

15. W. Winovich, W.C.A. Carlson, ISA Transactions, 19(2), 75 (1980).

16. R.C. Eschenbach, Proc. Workshop on Industrial Plasma Applic. M.I. Boulos ed., Pugnochiuso, Italy, 127 (1989).

17. S.L. Camacho, J. Dochterman, ibid., 9.

18. T. Shinoda, et al., ibid, 61.

19. N.A. Bareza, T.K. Curr, G.M. Denton, ibid, 115.

20. S.D. Savkar, P.A. Siemers, ibid, 80.

21. C. Li, B.W. Yu, S.L. Girshick, Proc. 10th ISPC, Bochum, (1991).

22. T. Yoshida, T. Tani, H. Nishimara, K. Akashi, J. Appl. Phys., 54,640 (1983).

23. Y. Mitsuda, T. Yoshida, K. Akashi, Rev. Sci. Instrum., 60, 249 (1989).

24. M. Moisan, R. Pantel, J. Hubert, Contrib. Plasma Phys., 80, 293 (1990). 